\title{
Quantitative Electron Probe Microanalysis at Low and Multiple Voltages
}

\author{
X. Llovet, * and C. Merlet** \\ * SCT, Universitat de Barcelona. Lluís Solé i Sabarís, 1-3. ES-08028 Barcelona. Spain. \\ ** GM, CNRS, Université de Montpellier II, Pl. E. Bataillon. FR-34095 Montpellier Cedex 5. \\ France.
}

Electron probe microanalysis (EPMA) is an analytical technique conventionally used to determine quantitatively the chemical composition of solid materials on the micrometer scale. Recently, with the incorporation of field-emission electron sources in EPMA equipment, electron probes with small diameters that deliver relatively high currents $(<100 \mathrm{nA})$ at low beam energies $(5-10 \mathrm{keV})$ have become available. This has opened the possibility of obtaining x-ray maps of chemical contrast and point analyses with sub-micrometer lateral resolution. However, EPMA at low beam energies is accompanied by experimental and analytical problems which may affect the accuracy of quantitative results (see e.g. [1]). First of all, the less intense, low-energy x-ray lines (mostly L- and M-lines) have to be used, which are often affected by peak shifts, peak overlapping and larger uncertainties in the mass-attenuation coefficients (MAC). Moreover, at low beam voltages, a surface layer of several nanometers in thickness represents a much larger fraction of the sample and, therefore, the influence of carbon contamination, surface oxidation, the quality of the sample polish or a conductive coating becomes more significant. To overcome these difficulties, new analytical strategies are required. The possibility of using low and multiple beam energies along with the development of thin-film correction programs, has also stimulated the use of EPMA for the characterization of thin films and multilayers, with thicknessess in the sub-micrometer range [2]. Measurements at multiple voltages provide the information required to separately characterize a layer or multiple layers on a substrate, which are in turn useful to improve the accuracy of EPMA at low voltage. However, the accuracy of thin film analysis by EPMA, which mainly relies on that of the analytical models underlying the thin-film programs, is still not firmly established.

In this communication, we illustrate the possibilities and limitations of EPMA at low and multiple voltages for the analysis of features with sub-micrometer dimensions. As a case example, we discuss the results and problems associated with the analysis of alloy steel at low voltage [3]. Three alloy steel samples were prepared from ingots of the corresponding alloys and treated so as to obtain an homogeneous structure. The (global) composition of the samples was obtained by X-Ray Fluorescence, Spark Optical Emission Spectroscopy and LECO analysis and was in the following range (in wt.\%): $\mathrm{N} \mathrm{0.02-0.05,} \mathrm{Si} \mathrm{0.48-1.34,} \mathrm{Cr} \mathrm{17.18-28.34,} \mathrm{Mn} \mathrm{0.74-1.61,} \mathrm{Fe} \mathrm{25.72-66.51,} \mathrm{Co}$ 0.03-0.34, $\mathrm{Ni} 11.24-39.75, \mathrm{Cu} 0.31-1.54$, Mo 0.40-3.11. EPMA analyses of the samples were conducted in different laboratories using different instrumentation and methodologies, with the only requirement to perform the analyses at a beam energy of $6 \mathrm{keV}$. The results showed a systematic underestimation of the Cr content, with relative errors ranging from $-8 \%$ to $-19 \%$, and an overestimation of $\mathrm{Fe}$ and $\mathrm{Ni}$, with relative errors ranging from +0.5 to $+7 \%$, and from $+15 \%$ to $+30 \%$, respectively. To elucidate the reasons for the differences observed and the scatter of results, one set of the analyses was recalculated using different matrix-correction procedures, different MACs and different standards. Only when the standards used were also alloy steel samples, the 
results were close to the nominal compositions. The significant dependence of the MACs pertaining to the L-lines of elements such as Fe, $\mathrm{Cr}$ and $\mathrm{Ni}$ on chemical bonding [4] is most likely the main source of uncertainty in this example.

Finally, we assess the accuracy of thin-film programs (e.g. STRATAGEM, XFILM) and Monte Carlo (MC) simulation codes (e.g. PENELOPE) widely employed for the analysis at multiple voltages of thin films and multilayers with thicknesses in the sub-micrometer range. To this end, we compare the predictions of the mentioned programs with experimental k-ratios from wellcharacterized thin film samples available in the literature (e.g. [5]). The results indicate that the analytical models and MC programs perform quite differently on the same dataset. The most sophisticated MC program PENELOPE [6] gives the closest correspondence with experiment but still shows a tendency to underestimate experimental $\mathrm{k}$-ratios by $10 \%$ for films that are thin compared to the electron range [7].

\section{References}

[1] P. Willich and R. Bethke, Mikrochim. Acta [Suppl.] 13 (1996) 631.

[2] X. Llovet and C. Merlet, Micros. Microanal. 16 (2010) 21.

[3] X. Llovet et al. Proceedings of EMAS 2011, Angers (France), 2011.

[4] J.L.Pouchou, Mikrochim. Acta [Suppl.] 13 (1996) 39.

[5] G.F. Bastin and H.J.M. Heijligers, X-Ray Spectrom. 29 (2000) 212.

[6] P.Statham, X.Llovet and P.Duncumb, Proceedings of EMAS 2011, Angers (France), 2011

[7] F. Salvat et al., PENELOPE. A Code System for Monte Carlo Simulation of Electron and Photon Transport, OECD/Nuclear Energy Agency, Issy-les-Moulineaux, France, 2009.

[8] Acknowledgement: This work was partially supported by the Spanish Ministerio de Ciencia y Innovación, project no. FPA2009-14091. 